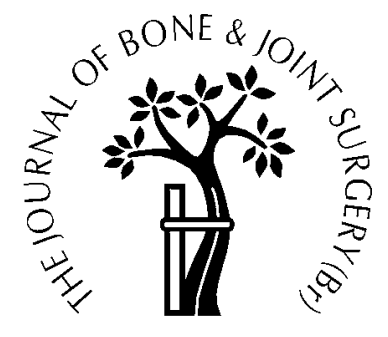

\title{
Chronic osteomyelitis
}

\section{THE EFFECT OF THE EXTENT OF SURGICAL RESECTION ON INFECTION-FREE SURVIVAL}

\author{
A. H. R. W. Simpson, M. Deakin, J. M. Latham \\ From the Nuffield Department of Orthopaedic Surgery, Oxford, England
}

W e studied prospectively a consecutive series of 50 patients with chronic osteomyelitis. Patients were allocated to the following treatment groups: 1) wide resection, with a clearance margin of $5 \mathrm{~mm}$ or more; 2) marginal resection, with a clearance margin of less than $5 \mathrm{~mm}$; and 3 ) intralesional biopsy, with debulking of the infected area. All patients had a course of antibiotics, intravenously for six weeks followed by orally for a further six weeks. No patients in group 1 had recurrence. In patients treated by marginal resection (group 2), 8 of 29 (28\%) had recurrence. All patients who had debulking had a recurrence within one year of surgery. We performed a survival analysis to determine the time of the recurrence of infection. In group 2 there was a higher rate of recurrence in type-B hosts $(p<0.05)$; no type-A hosts had recurrence. This information is of use in planning surgery for chronic osteomyelitis.

J Bone Joint Surg [Br] 2001;83-B:403-7.

Received 4 November 1999; Accepted after revision 10 October 2000

The management of chronic osteomyelitis continues to pose a major challenge for orthopaedic surgeons. Throughout the world chronic osteomyelitis is most commonly of haematogenous origin. In countries where effective antibiotics are administered in the early stages of acute osteomyelitis, the incidence of chronic osteomyelitis has decreased. In these countries it occurs after trauma in relation either to open fractures or to internal fixation.

Since the work of Stark ${ }^{1}$ over 50 years ago, the emphasis of the surgical management of chronic osteomyelitis has been on radical excision of bone to achieve control of the

A. H. R. W. Simpson, DM, FRCS, Professor of Orthopaedic Surgery Department of Orthopaedic Surgery, Princess Margaret Rose Orthopaedic Hospital, Frogston Road West, Edinburgh EH10 7ED, UK.

M. Deakin, MS, FRCS, Specialist Registrar

J. M. Latham, MA, MCh, FRCS Orth, Consultant Orthopaedic Surgeon Nuffield Orthopaedic Centre, Windmill Road, Headington, Oxford OX3 7LD, UK

Correspondence should be sent to Professor A. H. R. W. Simpson.

(C)2001 British Editorial Society of Bone and Joint Surgery 0301-620X/01/310727\$2.00 infection. Aggressive skeletal debridement, however, often leads to significant segmental defects which require complex reconstruction, with prolonged treatment times and significant morbidity. ${ }^{2-4}$ A decision to perform such extensive surgery should therefore be based on the likely duration of treatment and the possible morbidity, and also on a knowledge of the likelihood of achieving control of infection. To our knowledge, the effect of the extent of surgery on the incidence of recurrence of infection in patients with chronic osteomyelitis has not been investigated.

We therefore studied, prospectively, a consecutive series of 50 patients with chronic osteomyelitis treated by either wide resection of bone, by marginal resection, or by intralesional biopsy, in order to determine the effectiveness of the control of infection in these different treatment groups.

\section{Patients and Methods}

There were 39 men and 11 women with a mean age at presentation of 49 years (13 to 82). The diagnosis of chronic osteomyelitis was made on the basis of clinical presentation and imaging, and confirmed bacteriologically and histologically from intraoperative specimens. The aetiology of the chronic osteomyelitis was post-traumatic in 31 patients $(62 \%)$, haematogenous in $12(24 \%)$ and by spread from an overlying ulcer in seven (14\%).

Both systemic and local factors may influence the development and natural history of chronic osteomyelitis. We classified our patients as type A or type B according to the system of Cierny and Mader. "In type A there were no adverse factors. The factors categorising a patient as type B and the number of patients with specific factors are shown in Table I.

Depending on the anatomical location of disease, the extent of the osteomyelitis, the patients' age and comorbidity, and their preference of treatment options, a decision was made about surgical intervention. The patients were prospectively classified at the time of surgery into the three groups by the senior author (AHRWS).

Group 1 (wide excision). All necrotic and infected bone was excised with $5 \mathrm{~mm}$ or more of clearance. The remaining bone was clearly viable with good punctate bleeding.

Group 2 (marginal excision). All necrotic and infected bone was excised, but with less than $5 \mathrm{~mm}$ of clearance. 
Table I. Systemic and local factors affecting the response of infection as suggested by Cierny and Mader. Type-B categories observed in our study are in bold, with the numbers of patients with the factors in parentheses

\begin{tabular}{ll}
\hline Systemic (type B) & Local (type B) \\
\hline Malnutrition & Chronic lymphoedema \\
Renal, hepatic failure & Venous stasis \\
Diabetes mellitus (3) & Major vessel compromise \\
Chronic hypoxia & Arteritis \\
Immune disease & Extensive scarring \\
Malignancy (1) & Radiation fibrosis \\
Extremes of age & Small vessel disease \\
Immunosuppression or immune deficiency (3) & \\
Tobacco abuse (> two packs of cigarettes per day) (13) & \\
\hline
\end{tabular}

Further excision of bone would have necessitated segmental excision and therefore considerably increased the morbidity. In some cases there were areas where the bone bleeding was uncertain.

Group 3 (intralesional biopsy). In patients in whom the benefits of surgery were felt to be outweighed by the potential morbidity of the treatment, pus was drained, infected tissue debulked, small sequestra removed and a thorough lavage performed.

In all cases sufficient cortex was removed to allow a good view of the bone, which was possible through a unicortical window in cases in groups 2 and 3, but more commonly involved more extensive removal of cortical bone. There were $15(30 \%)$ patients in group 1, $29(58 \%)$ in group 2 and six (12\%) in group 3. All wound edges and sinus tracks were excised and the adjacent soft tissue was resected back to tissue that bled briskly.

All patients had MRI studies as part of the preoperative planning to determine the likely extent of bone resection. When there was a normal medullary fat signal the adjacent cortical bone was considered to be healthy. MRI was less useful in patients in whom there were areas of abnormal signal or artefact from previous metal implants. The actual extent of bone resection was determined at the time of surgery. MRI was also of value in delineating latent extraosseous collections of pus. If the soft-tissue defect exceeded the predicted bone defect, the patients were also assessed before surgery by a plastic surgeon.

At operation five specimens of tissue were collected, each with separate instruments, and sent for microscopy, aerobic and anaerobic culture, and for analysis of antibiotic sensitivity. Further specimens were sent for histological examination to confirm that the appearance was consistent with osteomyelitis, and to look for organisms.

Obliteration of dead space after debridement was achieved by reconstructive procedures involving either bone (shortening, corticotomy and distraction) or soft tissues (local myoplasties or free flaps), or direct skin closure with gentamicin-impregnated polymethylmethacrylate beads placed in the defect, but protruding through the wound. These beads were gradually removed over the ensuing two weeks to allow granulation tissue to fill the defect.
After surgery broad-spectrum antibiotics were administered intravenously to all patients. This was modified after the results of cultures and sensitivities were obtained and continued for six weeks. With those antibiotics for which the toxic level was near the treatment range, the serum levels were checked weekly. In most patients intravenous treatment was carried out on an outpatient basis supervised by a home intravenous therapy team. The patients were thereafter given antibiotics orally for six weeks.

All patients were followed up regularly, with a mean length of follow-up of 26.2 months (12 to 48). Recurrence of infection was diagnosed by local signs and symptoms of infection, sinus formation or drainage, a raised level of Creactive protein or ESR, or of systemic symptoms such as a fever for which no other cause could be found.

\section{Results}

The clinical details and the outcome of treatment for all patients are given in Table II.

There were no recurrences in patients in group 1, whereas eight of $29(28 \%)$ in group 2 had recurrence of infection. The difference in the rate of recurrence between the two groups was statistically significant (chi-squared test, $\mathrm{p}<0.05)$.

A comparative analysis of variables previously reported to affect the rate of recurrence, including age, the aetiology and the site of the osteomyelitis, the Cierny and Mader classifications, ${ }^{5,6}$ (Table I) the host status, ${ }^{7}$ the duration of the osteomyelitis and causative organisms showed no significant differences between groups 1 and 2. Therefore, it is likely that the differences in rates of recurrence are a true reflection of the effect of the extent of surgery.

Despite accurate microbiological diagnosis from intralesional biopsy and of appropriate antibiotic therapy for three months, all patients in group 3 had a recurrence of infection within the first year of follow-up (Table II). A statistically significant difference in the rate of recurrence was evident in all three treatment groups (chi-squared test, $p<0.05$ ). To highlight the effect of treatment on the rate of recurrence, a 'survival analysis' was performed to illustrate the disease-free intervals for the three groups (Fig. 1). 
Table II. Details of the 50 patients treated for chronic osteomyelitis

\begin{tabular}{|c|c|c|c|c|c|c|c|c|c|c|c|c|}
\hline Patient & $\begin{array}{l}\text { Age } \\
(\mathbf{y r})\end{array}$ & $\begin{array}{l}\text { Treatment } \\
\text { group }\end{array}$ & Aetiology* & $\begin{array}{l}\text { Site of } \\
\mathrm{OM}_{\dagger} \dagger\end{array}$ & $\begin{array}{l}\text { CM } \\
\text { class }\end{array}$ & $\begin{array}{l}\text { Host } \\
\text { status }\end{array}$ & $\begin{array}{l}\text { Duration } \\
\text { of OM } \dagger \\
\text { (yrs) }\end{array}$ & $\begin{array}{l}\text { Skeletal } \\
\text { stabilisation }\end{array}$ & $\begin{array}{l}\text { Dead space } \\
\text { management }\end{array}$ & Organisms§ & $\begin{array}{l}\text { Follow-up } \\
\text { (mths) }\end{array}$ & $\begin{array}{l}\text { Recurrent } \\
\text { infection } \\
\text { (mths) }\end{array}$ \\
\hline 1 & 47 & 1 & PT & Femur & III & A & 2 & No & Gent. beads & MRSA & 48 & No \\
\hline 2 & 57 & 1 & $\mathrm{PT}$ & Tibia & IV & A & 1 & Ex fixation & Free flap & CNS & 18 & No \\
\hline 3 & 51 & 1 & $\mathrm{PT}$ & Tibia & II & A & 5 & No & Gent. beads & Multiple & 15 & No \\
\hline 4 & 62 & 1 & PT & Femur & III & A & 31 & No & Gent. beads & Staph. aureus & 22 & No \\
\hline 5 & 25 & 1 & PT & Femur & IV & B & 1 & Ex fixation & Gent. beads & Multiple & 13 & No \\
\hline 6 & 44 & 1 & PT & Tibia & I & A & 3 & No & Free flap & Staph. aureus & 36 & No \\
\hline 7 & 31 & 1 & PT & Humerus & III & A & 5 & Plate & Free fibula & Diphtheroids & 29 & No \\
\hline 8 & 53 & 1 & PT & Femur & IV & B & 30 & No & Amputation & Multiple & 20 & No \\
\hline 9 & 58 & 1 & PT & Femur & IV & B & 24 & Ex fixation & Gent. beads & Multiple & 17 & No \\
\hline 10 & 47 & 1 & PT & Femur & III & A & 17 & No & Local flap & Multiple & 13 & No \\
\hline 11 & 49 & 1 & PT & Femur & IV & A & 1 & Ilizarov & Gent. beads & MRSA & 26 & No \\
\hline 12 & 64 & 1 & PT & Femur & III & B & 44 & No & Gent. beads & Multiple & 48 & No \\
\hline 13 & 28 & 1 & $\mathrm{H}$ & Radius & III & A & 1 & No & Gent. beads & Multiple & 35 & No \\
\hline 14 & 15 & 1 & $\mathrm{H}$ & Tibia & III & A & 1 & No & Gent. beads & Staph. aureus & 26 & No \\
\hline 15 & 78 & 1 & $\mathrm{H}$ & Tibia & IV & A & 60 & No & Amputation & Pseudomonas & 38 & No \\
\hline 16 & 58 & 2 & PT & Calcaneum & III & B & 1 & No & Gent. beads & Multiple & 16 & No \\
\hline 17 & 62 & 2 & PT & Femur & III & A & 7 & No & Gent. beads & Staph. aureus & 34 & No \\
\hline 18 & 32 & 2 & PT & Tibia & III & B & 19 & No & Gent. beads & CNS & 40 & No \\
\hline 19 & 51 & 2 & PT & Tibia & III & A & 11 & No & Free flap & Multiple & 15 & No \\
\hline 20 & 38 & 2 & PT & Tibia & I & A & 2 & No & Gent. beads & Multiple & 41 & No \\
\hline 21 & 18 & 2 & PT & Tibia & II & A & 4 & No & Gent. beads & Multiple & 16 & No \\
\hline 22 & 28 & 2 & PT & Tibia & III & A & 9 & No & Gent. beads & CNS & 12 & No \\
\hline 23 & 21 & 2 & PT & Tibia & III & A & 2 & No & Gent. beads & Staph. aureus & 17 & No \\
\hline 24 & 28 & 2 & PT & Femur & III & A & 8 & No & Gent. beads & Multiple & 33 & No \\
\hline 25 & 58 & 2 & $\mathrm{H}$ & Calcaneum & III & B & 1 & No & Gent. beads & Diphtheroids & 22 & No \\
\hline 26 & 79 & 2 & $\mathrm{H}$ & Femur & III & B & 5 & No & Gent. beads & Staph. aureus & 48 & No \\
\hline 27 & 32 & 2 & $\mathrm{C}$ & Femur & III & B & 2 & No & Gent. beads & Multiple & 25 & No \\
\hline 28 & 56 & 2 & $\mathrm{H}$ & Humerus & III & A & 3 & No & Gent. beads & Staph. aureus & 14 & No \\
\hline 29 & 55 & 2 & $\mathrm{H}$ & Humerus & III & A & 1 & No & Gent. beads & CNS & 30 & No \\
\hline 30 & 39 & 2 & $\mathrm{C}$ & Metatarsal & II & A & 5 & No & None & Staph. aureus & 36 & No \\
\hline 31 & 13 & 2 & $\mathrm{H}$ & Calcaneum & III & A & 1 & No & Gent. beads & Staph. aureus & 12 & No \\
\hline 32 & 60 & 2 & $\mathrm{C}$ & Phalanx & III & B & 2 & No & None & $\beta$-haem. strep & 17 & No \\
\hline 33 & 35 & 2 & $\mathrm{H}$ & Tibia & I & A & 1 & No & Gent. beads & Staph. aureus & 48 & No \\
\hline 34 & 27 & 2 & $\mathrm{H}$ & Tibia & III & A & 1 & No & Gent. beads & Staph. aureus & 22 & No \\
\hline 35 & 66 & 2 & $\mathrm{H}$ & Femur & IV & B & 23 & Ex fixation & Gent. beads & Multiple & 14 & No \\
\hline 36 & 27 & 2 & $\mathrm{C}$ & Tibia & III & B & 3 & No & Gent. beads & Multiple & 26 & No \\
\hline 37 & 33 & 2 & PT & Humerus & III & B & 6 & No & Gent. beads & Multiple & Recurrence & 0 to 3 \\
\hline 38 & 52 & 2 & PT & Femur & III & B & 5 & No & Gent. beads & Staph. aureus & Recurrence & 3 to 6 \\
\hline 39 & 50 & 2 & PT & Tibia & III & B & 28 & No & Gent. beads & CNS & Recurrence & 3 to 6 \\
\hline 40 & 40 & 2 & PT & Humerus & III & B & 10 & No & Gent. beads & Staph. aureus & Recurrence & 6 to 9 \\
\hline 41 & 78 & 2 & PT & Tibia & III & B & 27 & No & Gent. beads & Staph. aureus & Recurrence & 21 to 24 \\
\hline 42 & 75 & 2 & PT & Femur & III & B & 36 & No & Gent. beads & Strep. faecalis & Recurrence & 3 to 6 \\
\hline 43 & 75 & 2 & $\mathrm{H}$ & Calcaneum & III & B & 3 & No & Gent. beads & Staph. aureus & Recurrence & 3 to 6 \\
\hline 44 & 51 & 2 & $\mathrm{C}$ & Calcaneum & III & B & 2 & No & Gent. beads & Multiple & Recurrence & 6 to 9 \\
\hline 45 & 82 & 3 & PT & Femur & IV & $\mathrm{C}$ & 15 & - & - & Multiple & Recurrence & 0 to 3 \\
\hline 46 & 75 & 3 & PT & Tibia & III & $\mathrm{C}$ & 13 & - & - & Staph. aureus & Recurrence & 3 to 6 \\
\hline 47 & 57 & 3 & $\mathrm{PT}$ & Tibia & IV & $\mathrm{C}$ & 37 & - & - & Proteus & Recurrence & 0 to 3 \\
\hline 48 & 70 & 3 & PT & Femur & III & $\mathrm{C}$ & 42 & - & - & Staph. aureus & Recurrence & 3 to 6 \\
\hline 49 & 76 & 3 & PT & Calcaneum & III & $\mathrm{C}$ & 5 & - & - & Staph. aureus & Recurrence & 9 to 12 \\
\hline 50 & 42 & 3 & $\mathrm{C}$ & Pelvis & IV & $\mathrm{C}$ & 4 & - & - & Multiple & Recurrence & 0 to 3 \\
\hline
\end{tabular}

* PT, post-traumatic; $\mathrm{H}$, haematogenous; $\mathrm{C}$, contiguous

$\dagger$ osteomyelitis

\$ Cierny and Mader ${ }^{5,6}$ classification of chronic osteomyelitis

$\S$ CNS, coagulase-negative staphylococcus; MRSA, methicillin-resistant Staph. aureus 


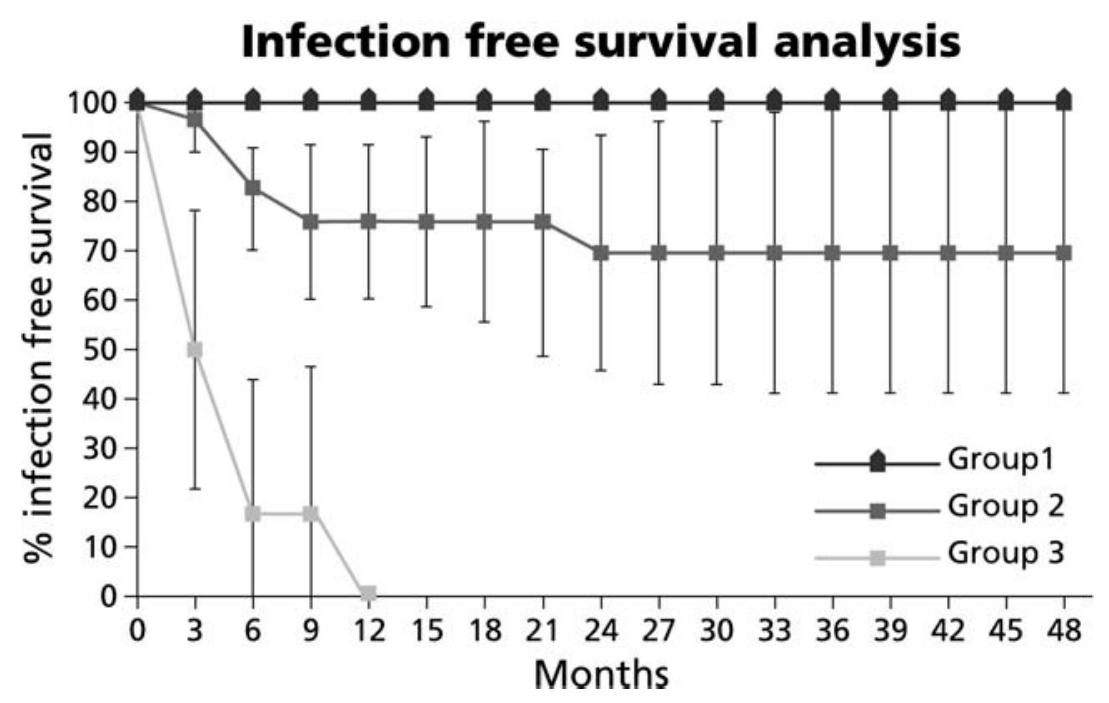

Fig. 1

Cumulative infection-free period for patients with chronic osteomyelitis treated by wide resection, marginal resection or intralesional debulking.

Patients in group 2 were further analysed to determine factors which might affect the likelihood of recurrent infection. The factors studied included the aetiology and the site of the osteomyelitis, the Cierny and Mader class of osteomyelitis, host comorbidity, the duration of the infection and the causative organisms. Those factors found to be significantly related to the recurrence of infection were comorbidity $^{7}$ and the duration of osteomyelitis before surgical intervention. Patients with a normal physiological response to infection and surgery were designated class-A hosts. Compromised patients with local, systemic or combined deficiencies of wound healing were considered class-B hosts. In patients in group 2 without recurrence of infection, $13(62 \%)$ were type-A hosts and eight (38\%) were type-B hosts. All eight patients with recurrent infection were type-B hosts. This difference was statistically significant (chi-squared test with correction of continuity, $\mathrm{p}<0.01)$.

The duration of osteomyelitis was determined as the time from the first onset of symptoms to the time of surgical intervention. For patients in group 2 in whom no recurrence of infection occurred, the mean duration of osteomyelitis was 5.3 years ( 1 to 23 ). For those with recurrent infection, it was 14.6 years ( 2 to 36 ). This difference was significant (Student's $t$-test, $\mathrm{p}<0.02$ ).

\section{Discussion}

It is essential to remove all necrotic and infected bone when treating osteomyelitis. Although the importance of this principle is stressed in the relevant literature, the method of determining the excision margins is often not stated. ${ }^{1,8}$ Many techniques have been used to determine whether bone is alive or dead, including non-vital dye, ${ }^{9}$ laser-
Doppler flowmetry ${ }^{10}$ and the radiological appearance described as sclerosis. ${ }^{11}$ The technique most commonly used, however, is the appearance of the bone at the time of surgery, and the presence of punctate bleeding ('paprika sign'). ${ }^{2,7,12-14}$ We used this technique to assess whether or not the bone was viable. In all three groups appropriate antibiotics were given for six weeks intravenously and then orally for six weeks. Despite this chemotherapeutic regime with antibiotic delivered parenterally for a prolonged period of time and accurate monitoring to ensure that the levels were in the therapeutic range, all patients in group 3 had recurrence of osteomyelitis. This suggests that even with modern chemotherapeutic regimes, the degree of surgical clearance remains critical.

Survival analysis is commonly used, especially in oncology, to describe the number of recurrences of disease, and also the length of time that the disease had remained under control. We used calculations from the survival table to provide information about the number of recurrent infections and the length of time that the various types of surgical clearance provided control of the infection. This approach has also been used to analyse a mixed series of acute and chronic infections ${ }^{15}$ and found to be useful. Most of the infections recurred within one year of surgery, which is usual. ${ }^{15}$ Of our 14 patients who suffered a recurrence, however, in one this was late, 21 months after surgery. In the 44 patients who had a wide or marginal resection, there were eight (18\%) recurrences. Swiontkowski et al ${ }^{16}$ reported a recurrence rate of $9 \%$ in adult cases of osteomyelitis, but their follow-up ranged from 0 to 62 months and they did not state the host category. Some of these patients had an infected nonunion and would normally be treated with a wide resection. Kelly ${ }^{10}$ reported a rate of success of $86 \%$ in femoral and tibial nonunions followed up for two years, 
and Cierny ${ }^{17}$ reported a success rate of $93 \%$ in infected nonunion in patients in whom all necrotic and ischaemic tissue had been excised. There was a minimum follow-up of five years without any recurrences at two years, which is similar to the results of our group undergoing wide resection.

The duration of the infective process also adversely affected the prognosis. It has been suggested that prolonged infection causes increased sclerosis and scarring of the softtissue envelope, making the infection more resistant to treatment. ${ }^{18}$ Alternatively, it is possible that these patients had disease which had not been eradicated before attending the tertiary referral centre, either because they had hidden areas of disease or because the causative bacteria were difficult to culture or treat.

The local or systemic immune response of some patients may be suboptimal which may lead to higher recurrence rate. ${ }^{10} \mathrm{We}$ found that the type of host had less bearing on the outcome when patients had a wide (group 1) rather than a marginal excision (group 2). In group 2, significantly more of the type-B hosts had recurrence of infection (50\%) compared with type A $(0 \%)$. Walenkamp ${ }^{14}$ has suggested that there is a gradual transition between dead and viable bone. Some of the specimens taken in this study showed the bone to be partly necrotic and partly viable, which would support his hypothesis. It is possible that type-A hosts are able to resorb a microscopic amount of non-viable bone more quickly when compared with type-B hosts. This could then account for the difference in the rates of recurrence observed with the different types of host.

We used the same principles of treatment for patients with infected nonunion. As these patients do not have skeletal integrity, it is not possible to carry out a marginal procedure and leave the patient with an intact bone. A wide clearance of the bone was therefore carried out in all patients with infected nonunion.

In planning the management of patients with chronic osteomyelitis it is important to take into account the morbidity that the disease is causing, the type of host, and the magnitude of the proposed surgical procedures. Our study provides valuable information on the prognosis after surgical procedures of different magnitude in different hosts. As a result of this study we have altered our practice and are now more likely to carry out marginal excision procedures, especially in type-A hosts. In determining the optimum management for a patient with chronic osteomyelitis the data from our study should be of value.

No benefits in any form have been received or will be received from a commercial party related directly or indirectly to the subject of this article.

\section{References}

1. Stark WJ. The use of pedicled muscle flaps in the surgical treatment of chronic osteomyelitis resulting from compound fractures. J Bone Joint Surg [Br] 1946;28-B:343-50.

2. May JW, Jupiter JB, Weiland AJ, Byrd HS. Clinical classification of post-traumatic tibial osteomyelitis. J Bone Joint Surg [Am] 1989;71-A:1422-8.

3. Papineau L-J, Alfageme A, Dalcourt JP, Pilou L. Osteomyelite chronique: excision et grette de spongieux a l'air libre apres mises a plat extensives. Int Orthop 1979;3:165-76.

4. Wood MB, Cooney WP III. Vascularised bone segment transfers for management of chronic osteomyelitis. Orthop Clin North Am 1984;15:461-47.

5. Cierny G III, Mader JT. Adult chronic osteomyelitis. Orthopaedics 1984;7;10:1557-64.

6. Cierny G. Chronic osteomyelitis: results of treatment. Inst Course Lect 1990;39:495-508.

7. Mader JT, Cripps MW, Calhoun JH. Adult post-traumatic osteomyelitis of the tibia. Clin Orthop 1999;360:14-21.

8. Cierny G, Mader JT, Pennincck JJ. A clinical staging system for adult osteomyelitis. Contemp Orthop 1985;10:17-37.

9. Koval KJ, Meadows SE, Rosen H, Silver L, Zucherman JD. Posttraumatic tibial osteomyelitis: a comparison of three treatment approaches. Orthopedics 1992;15:455-60.

10. Kelly PJ. Infected nonunion of the femur and tibia. Clin Orthop North Am 1985;15:481-90.

11. Hass DW, McAndrew MP. Bacterial osteomyelitis in adults: evolving considerations in diagnosis and treatment. Am J Med 1996;101:550-61.

12. McNally MA, Small JO, Tofighi HG, Mollan RAB. Two stage management of chronic osteomyelitis of the long bones: the Belfast technique. J Bone Joint Surg [Br] 1993;75-B:375-80.

13. Rowling DE. The positive approach to chronic osteomyelitis. $J$ Bone Joint Surg [Br] 1959;41-B:681-8.

14. Walenkamp GH. Chronic osteomyelitis. Acta Orthop Scand 1997; 68:497-506.

15. Walenkamp GH, Kleijn LL, Leeuw M. Osteomyelitis treated with gentamicin-PMMA beads: 100 patients followed for 1-12 years. Acta Orthop Scand 1998;69:518-22.

16. Swiontkowski MF, Hanel DP, Bedder NB, Schwappach JR. A comparison of short- and long-term intravenous antibiotic therapy in the postoperative management of adult osteomyelitis. J Bone Joint Surg [Br] 1999;81-B:1046-50.

17. Cierny G. Infected tibial nonunions (1981-1995): the evolution of change. Clin Orthop 1999;360:97-105.

18. West WF, Kelly PJ, Martin WJ. Chronic osteomyelitis: factors affecting the results of treatment in 186 patients. JAMA 1970;213:1837-42. 